\title{
Early antibiotic administration prevents cognitive damage induced by pneumococcal meningitis in Wistar rats
}

\author{
Tatiana Barichello*1, Clarissa M Comim² , Geruza Z Silva ${ }^{1}$, Andréa C Ramos ${ }^{1}$, \\ Ana Lúcia S Batista ${ }^{1}$, Geovana D Savi ${ }^{1}$, Gustavo Feier ${ }^{2}$, Felipe Dal-Pizzol ${ }^{1}$ and \\ João Quevedo
}

\author{
Address: ${ }^{1}$ Laboratório de Fisiopatologia Experimental, Universidade do Extremo Sul Catarinense, 88806-000 Criciúma, SC, Brazil and \\ ${ }^{2}$ Laboratório de Neurociências, Programa de Pós-Graduação em Ciências da Saúde, Unidade Acadêmica de Ciências da Saúde, Universidade do \\ Extremo Sul Catarinense, 88806-000 Criciúma, SC, Brazil \\ Email: Tatiana Barichello* - tba@unesc.net \\ * Corresponding author
}

from Infectious diseases of the nervous system: pathogenesis and worldwide impact Paris, France. 10-13 September 2008

Published: 23 September 2008

BMC Proceedings 2008, 2(Suppl I):P5

This abstract is available from: http://www.biomedcentral.com/I753-656I/2/SI/P5

(c) 2008 Barichello et al; licensee BioMed Central Ltd.

Bacterial meningitis is an infection of the central nervous system characterized by a strong inflammation of the meninges and the subarachnoid space. Pneumococcal meningitis in humans is associated with long-term sequelae including sensory-motor deficits, seizures, and impairments of learning and memory. In order to evaluate this in an animal model, Streptococcus pneumoniae was cultured overnight in Todd Hewitt broth, diluted in fresh medium and grown to logarithmic phase, washed and resuspended in sterile normal saline $5 \times 10^{9} \mathrm{cfu} / \mathrm{ml}$. Meningitis was induced by inoculating $10 \mu \mathrm{L}$ of the $S$. pneumoniae suspension into the cisterna magna of the animals (rats, 60 days old, weighing 250-300 g) after removal of $10 \mu \mathrm{L}$ of cerebrospinal fluid (CSF). All surgical procedures and bacteria administrations were performed under anesthesia. Meningitis was documented by a quantitative culture of $5 \mu \mathrm{l}$ of CSF obtained by puncture of the cisterna magna at $8 \mathrm{~h}$ and $16 \mathrm{~h}$ after infection followed by the initiation of the antibiotic treatment (ceftriaxone $100 \mathrm{mg} / \mathrm{kg}$ bid). On day 10 , rats were submitted to a behavioural task. Habituation to an open field was carried out in an open arena divided into 9 equal rectangles by black lines. Animals were gently placed on the left quadrant, and was allowed to explore the arena for 5 min (training session) and 24 hrs later submitted again to a similar session (test session). Crossing of the black lines and rearing performed in both sessions were counted. All data are presented as mean \pm SD. Data were analyzed by Student's T test, considered $p<0.05$ to be significant. In the rats that were treated with antibiotic beginning at both $8 \mathrm{~h}$ and $16 \mathrm{~h}$ after infection no differences in the number of crossings and rearings were observed between groups in the habituation to the openfield training session ( $p>0,05)$. In the test session, in animals that antibiotic starts $8 \mathrm{~h}$ after infection, we did not observe reduction in both crossings and rearings in meningitis survivors rats compared with sham $(\mathrm{p}>0.05)$. However, in rats that antibiotic starts $16 \mathrm{~h}$ after infection, we observed a significant reduction in both crossings and rearings in meningitis survivors rats compared with sham $(\mathrm{p}<0.05)$. In conclusion, early antibiotic administration ( $8 \mathrm{~h}$ after infection) prevents cognitive damage induced by pneumococcal meningitis in Wistar rats.

Financial Support

CNPq, FAPESC and UNESC. 